\title{
Expert meeting report: towards a joint European roadmap to address the unmet needs and priorities of paediatric asthma patients on biologic therapy
}

\author{
Korneliusz Golebski ${ }^{1}$, Lente H.M. Dankelman ${ }^{1}$, Sofia Björkander ${ }^{2}$, Klaus Bønnelykke ${ }^{3}$, Paul Brinkman $\mathbb{D}^{1}$, \\ Antoine Deschildre $\mathbb{C}^{4}$, Yoni E. van Dijk ${ }^{1,5}$, Louise Fleming $\mathbb{1}^{6}$, Jonathan Grigg $\mathbb{C}^{7}$, Eckard Hamelmann ${ }^{8}$, \\ Simone Hashimoto (1) ${ }^{1,5}$, Michael Kabesch ${ }^{9}$, Susanna Klevebro ${ }^{2,10}$, Anke-Hilse Maitland-van der Zee ${ }^{1,5}$, \\ Simon K. Merid ${ }^{2}$, Antonio Nieto ${ }^{11}$, Jakob Niggel ${ }^{9}$, Caroline Nilsson $^{2,10}$, Uroš Potočnik ${ }^{12}$, Graham Roberts ${ }^{13}$, \\ Franca Rusconi $\mathbb{D}^{14}$, Sejal Saglani ${ }^{6}$, Elisangela Valente $\mathbb{D}^{9}$, Cornelis van Drunen ${ }^{15}$, Gang Wang ${ }^{2,16}$, \\ Erik Melén $\mathbb{B}^{2,10,17}$ and Susanne J.H. Vijverberg (D) ${ }^{1,5,17}$
}

\begin{abstract}
${ }^{1}$ Dept of Respiratory Medicine, Amsterdam UMC, University of Amsterdam, Amsterdam, The Netherlands. ${ }^{2}$ Dept of Clinical Science and Education, Södersjukhuset, Karolinska Institutet, Stockholm, Sweden. ${ }^{3}$ COPSAC, Copenhagen Prospective Studies on Asthma in Childhood, Herlev and Gentofte Hospital, University of Copenhagen, Copenhagen, Denmark. ${ }^{4} \mathrm{CHU}$ Lille, Pediatric Pulmonology and Allergy Unit, Hôpital Jeanne de Flandre, Université Nord de France, Lille, France. ${ }^{5}$ Dept of Pediatric Pulmonology, Emma Children's Hospital, Amsterdam UMC, Amsterdam, The Netherlands. ${ }^{6}$ Paediatric Respiratory Medicine, National Heart and Lung Institute, Imperial College and Royal Brompton Harefield NHS Foundation Trust, London, UK. ${ }^{7}$ Centre for Paediatrics, Blizard Institute, Queen Mary, University of London, London, UK. ${ }^{8}$ Klinik für Kinder and Jugendmedizin Kinderzentrum, Bethel Evangelisches Krankenhaus, Allergy Center, Ruhr University Bochum, Bielefeld, Germany. ${ }^{9}$ Dept of Pediatric Pneumology and Allergy, University Children's Hospital Regensburg (KUNO) at the Hospital St Hedwig of the Order of St John and the University Hospital, University of Regensburg, Regensburg, Germany. ${ }^{10}$ Sachs' Children and Youth Hospital, Södersjukhuset, Stockholm, Sweden. ${ }^{11}$ Children's Hospital La Fe, Pediatric Pulmonology and Allergy Unit, Instituto de Investigacion Sanitaria La Fe, Valencia, Spain. ${ }^{12}$ Center for Human Molecular Genetics and Pharmacogenomics, Faculty of Medicine, University of Maribor, Maribor, Slovenia. ${ }^{13}$ Department of Child Health, University of Southampton Hospital, Southampton, UK. ${ }^{14}$ Epidemiology Unit, Meyer Children's University Hospital, Florence, Italy. ${ }^{15}$ Department of Otorhinolaryngology, Amsterdam UMC, University of Amsterdam, Amsterdam, The Netherlands. ${ }^{16}$ Department of Integrated Traditional Chinese and Western Medicine, West China Hospital, Sichuan University, Sichuan, China. ${ }^{17}$ These authors contributed equally.
\end{abstract}

Corresponding author: Susanne J.H. Vijverberg (s.j.vijverberg@amsterdamumc.nl)

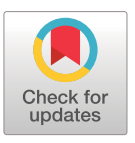

Copyright @The authors 2021

This version is distributed under the terms of the Creative Commons Attribution NonCommercial Licence 4.0. For commercial reproduction rights and permissions contact permissions@ersnet.org

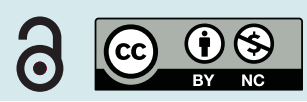

Shareable abstract (@ERSpublications)

A digital multidisciplinary European expert meeting took place on the 9 July 2020 to identify the unmet needs of paediatric severe asthma patients, and set the priorities for clinical and research activities ahead https://bit.ly/3CeLBHB

Cite this article as: Golebski K, Dankelman LHM, Björkander S, et al. Expert meeting report: towards a joint European roadmap to address the unmet needs and priorities of paediatric asthma patients on biologic therapy. ERJ Open Res 2021; 7: 00381-2021 [DOI: 10.1183/23120541.00381-2021].

Biologics use in severe paediatric asthma

The global prevalence of severe asthma among adolescents ranges from $4 \%$ to $11 \%$; and up to $7 \%$ of children with asthma display an uncontrolled and severe form that is often associated with a substantial burden on the quality of life of patients and their families, and increasing costs of healthcare [1, 2]. "Childhood asthma" is an umbrella term describing a heterogeneous disease comprising different phenotypes and a wide range of symptoms [3-5].

Despite decades of basic and clinical research, tailored strategies to modify the natural course of asthma, prevent severe exacerbations and inhibit lung function decline are still lacking. In addition, clinical phenotypes are only moderately reliable in the prediction of treatment responses and our current understanding of asthma endotypes is limited. Most asthma endotypes involve concomitant inflammatory pathways and distorted immune parameters. Advances in understanding severe paediatric asthma pathophysiological mechanisms and immunological pathways mediating the airway inflammation would allow better characterisation of these patients as well as optimised intervention, guided by treatable traits and biomarkers [6, 7]. 
This article has supplementary material available from openres.ersjournals.com

Received: 07 June 2021 Accepted: 28 July 2021
Recent studies have demonstrated the effectiveness of monoclonal antibodies (mAbs), also known as biologics, targeting type 2 inflammation in controlling the symptoms of severe asthma. Currently, four human mAbs are approved for use in children: mAbs that target interleukin (IL)-5 or IL-5 receptor (R) (mepolizumab and benralizumab), mAbs that target IL-4R (dupilumab), and mAbs that target immunoglobulin E (omalizumab). Omalizumab was the first biologic approved to treat moderate-to-severe allergic asthma ( $\geqslant 6$ years of age). Mepolizumab and dupilumab have been approved for severe eosinophilic asthma ( $\geqslant 6$ and $\geqslant 12$ years of age, respectively), while benralizumab has been approved in the USA to treat children ( $\geqslant 12$ years of age) with severe eosinophilic asthma [8-13].

The introduction of mAb agents in asthma treatment is a milestone in the application of personalised medicine. However, comparative studies and standardised algorithms for the management of paediatric severe asthma to guide the best therapeutic option for paediatric patients with severe asthma are lacking [14]. More personalised medicine approaches may benefit the patient by better matching patients with the most appropriate therapy. Risk stratification, remote monitoring and the integration of multiple data sources could help tailor management for the individual child with severe asthma.

A digital multidisciplinary European expert meeting took place on 9 July 2020. In this workshop, we brought together European respiratory/allergy paediatricians, immunologists, epidemiologists and basic scientists to identify the unmet needs of paediatric severe asthma patients, and set the priorities for clinical and research activities ahead. The participants discussed ongoing initiatives and knowledge gaps, and formulated proposals on how to address these challenges. In this report, we describe the main findings of this expert meeting.

\section{Current knowledge gaps and challenges}

Except for omalizumab, a major constraint to the optimal and consistent use of biologics in paediatric severe asthmatic is the limited evidence regarding the safety and efficacy of biologics in children $[15,16]$ (table S1). This especially holds true for biologics that entered the market recently. In the large phase 3 trials of these treatments, children were underrepresented and constituted only $1-6 \%$ of included patients [10, 17-20]. Although it might be challenging to recruit paediatric patients, assessing efficacy and safety in this population is essential since their immune system is under development and asthma progression is related to age and sex [21]. Studies that assessed the safety of omalizumab or mepolizumab in severe asthmatic children after 52 weeks of treatment did not observe any treatment-related severe adverse events $[9,22]$ but potential long-term effects remain unclear. Furthermore, the mepolizumab studies included very few children and adolescents. Finally, there are, to date, no data on biologics in the preschool-age severe asthma. How these treatments might impact underlying disease mechanisms is only partly clear and potential disease-modifying effects are not well understood. Since these expensive drugs may require lifelong administration [23], careful consideration is needed to assess which child is eligible for which biologic. A better understanding of paediatric asthma endotypes that can evolve over time might also lead to novel insights into whether biologics that previously showed no efficacy in adults could still be promising candidates for children [24].

The American Thoracic Society and the European Respiratory Society (ERS) define severe asthma as asthma that requires treatment with high-dose inhaled corticosteroids plus a second controller (and/or systemic corticosteroids) to prevent it from becoming "uncontrolled" or "which remains uncontrolled despite this therapy" [25]. Application of adult definitions and treatment guidelines may not always be appropriate; children with severe asthma have more atopy, less airflow limitation and less association with obesity [26]. The lack of clear definitions of "paediatric severe asthma" and "treatment response" were also identified as important hurdles. Moreover, there is an urgent need to formulate a definition of severe preschool asthma/wheeze. A consensus is needed both for research on biologics in the paediatric population as well as for uniform clinical management across Europe, in accordance with the Paediatric Investigational Plans of the European Medicines Agency. Moreover, the lack of research funding for paediatric studies on biologics and lack of scientific collaboration with other inflammatory disease areas where biologics have been applied in the paediatric population were identified as important challenges.

Lastly, the COVID-19 era has led to significant changes in how healthcare is accessed and provided [27]. This brings new challenges, as well as opportunities, for the management of children on biologics. There is now a stronger focus on remote care and monitoring, on the use and development of digital tools that might help with this, and on possible home administration of biologics. Self- or caregiver-administered injections make it possible for patients that live further away from a clinic to receive treatment more consistently, but also requires proper training, as well as careful remote monitoring for complications and to ensure good adherence [28]. 
Ongoing initiatives: bringing European experts together

The high socioeconomic burden of uncontrolled disease, and the lack of clear indicators for treatment choice and responses to administrated treatment, especially for biologics, underline the need for better characterisation of paediatric asthma phenotypes/endotypes and novel therapeutic options. Several ongoing studies and initiatives within European consortia address those needs (table S2). Hurdles in recruitment and underrepresentation of children in severe asthma studies provide an urgent need for collaborative efforts between European medical centres. Therefore, bringing together the data that are already available from existing consortia may be the first step towards more efficient characterisation of the disease and treatment options. Pan-European standardisation of inclusion and exclusion criteria for study participants and operating protocols, as well as an agreement on sample and data collection, would further provide better validity of outcomes and enable access to large biobanks.

There was consensus on the need for new collaborative trials, real-life cohorts and in vitro pre-clinical models with standardised protocols on applicable paediatric antiasthmatic drugs. An urgent update and uniformisation of treatment guidelines that take into account switching from one biologic to another, compatibility of more than one biologic, dose adjustment and long-term (side-)effects of these therapies was considered essential. This requires a development of specific paediatric tools for evaluating severity, level of control and response to treatment, and as well as data harmonisation.

Identified research priorities

Seven priority research areas were identified: 1) early identification of young asthma patients, including preschoolers, with a high risk of progression to severe disease; 2) exploring molecular mechanisms underlying poor response to treatment; 3) prediction of response to biologics; 4) understanding mechanistic differences underlying paediatric and adult severe asthma phenotypes; 5) long-term efficacy and safety; 6) understanding patient and caregiver perspectives on biologics use; and 7) harmonising (access to) biologics for asthmatic children treatment across Europe (table 1).

The early identification of asthmatic children at greatest risk of progression to severe disease requires longitudinal follow-up combined with in-depth pheno-endotyping [29]. Since various European initiatives are ongoing in this area, connecting different paediatric initiatives was identified as an important next step, in addition to linking with other ERS Clinical Research Collaborations with an adult focus, such as SHARP (focusing on severe asthma in adults) [30] and CADSET (focusing on lung function trajectories).

To predict which patients will respond to which biologics, ideally, comparative crossover clinical trials are needed. However, these kinds of trials are likely to be very challenging. Pragmatic and noninferiority trials, such as the recently initiated TREAT trial in the UK, which aims to compare the efficacy of mepolizumab versus omalizumab in reducing asthma attacks in children, might be more feasible [31]. Real-life efficacy data, combined with prospectively collected biomarkers, have the potential to provide additional valuable information [32, 33]. International and multidisciplinary collaboration is critical to reaching a consensus on response definitions (as is currently ongoing with the 3TR consortium), to recruit enough patients and to harmonise research protocols.

Understanding molecular mechanisms and immunological pathways are essential for the assessment of biomarker candidates for treatment response as well as an acceleration of the development of novel therapeutics. Successful modern research collaborations increasingly include scientists and clinicians of different expertise. Today, ex vivo translational models, including monolayer cultures, coculture systems or organ-on-chip lung models, provide an unprecedented opportunity to study in vitro effects of promising drugs. Introduction of novel technologies, i.e. single-cell sequencing or CRISPR-Cas9, enable a functional validation of potential biomarkers predicted by genome-wide association studies in ex vivo airway cell/ tissue models [34, 35].

Knowledge as to which asthmatic patients progress into severe adult asthma is lacking, although risk factors like atopy, multiple allergic comorbidities, impaired lung function, obesity and environmental exposures are well-described [29]. Severe disease may differ substantially between children and adults. For example, blood eosinophils or airway eosinophils might not equal T2 inflammation in children, while they often do in adults [36]. Upcoming results from initiatives and consortia such as VIRASTHMA2, COBRAPed, UBIOPRED paediatric cohorts and SysPharmPediA might be able to shed more light on paediatric endotypes of severe disease.

The patient and caregiver perspectives must also be heard, as beliefs about treatment are known to influence treatment satisfaction and adherence [37]. Qualitative studies and active collaborations between 


\section{TABLE 1 Identified research priorities}

Research priority Action steps

Identifying young patients at highest risk for progression to severe disease

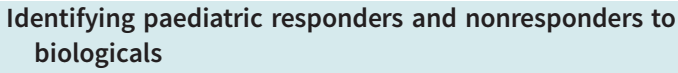

Understanding phenotypes of severe disease, and differences between adult and paediatric severe disease phenotypes

Long-term efficacy and safety of biologics use

Understanding molecular mechanisms to accelerate drug development using ex vivo translational models

\author{
Understanding patient and caregiver perspectives on \\ biologics use
}

Harmonising treatment protocols across Europe

\author{
Applying a precision medicine approach in severe paediatric \\ asthma
}

Establish a European real-world paediatric severe asthma cohort with longitudinal follow-up (into adulthood), building on existing initiatives and infrastructures (e.g. SPACE, UBIOPRED, GAN, PERMEABLE, COBRAPed, VIRASTHMA2 and the Danish National Database for Severe Asthma)

Standardise a definition of a responder/nonresponder

Large-scale collaboration to include paediatric patients with severe asthma/allergy

Collaborative study efforts using joint research/clinical protocols (e.g. pragmatic real-world studies and comparative studies with biologics)

Assess whether incorporation of identified predictive biomarkers in clinical decision-making models improve clinical outcomes and are cost-effective

In-depth phenotyping of severe asthma/allergy patients combining clinical characteristics and -omics data (e.g. UBIOPRED, COBRAPed, SysPharmPediA, SPACE, PERMEABLE and VIRASTHMA2) in combination with validation in adult cohorts (e.g. SHARP and UBIOPRED)

Long-term follow-up of severe paediatric asthma into adulthood

Establish a European real-world paediatric cohort with patients on biologics of choice and/or switchers with longitudinal follow-up (assess the maintenance dosage, long-term follow-up on overall health and QoL of patients)

Establish collaborations with immunologists and molecular biologists to ensure hypothesis/outcome validation in preclinical disease models

Implement the latest molecular biology techniques (i.e. CRISPR-Cas9 and single-cell sequencing assays) both to validate existing outcomes based on associations and to discover novel cell (sub)types that mediate underlying inflammatory processes

Develop noninvasive techniques to explore pathophysiological mechanisms

Perform qualitative studies on patient and caregiver experiences

Establish a European patient and caregiver advisory board specific for biologics use (in collaboration with European Lung Foundation and national patient organisations)

Develop and apply a platform for children and parents' involvement

Systematically assess which differences exist within the European countries

Develop online educational programmes and regularly update these programmes based on novel scientific evidence

Evaluate the added value of biomarker and -omics data for individual treatment selection

Combine disease history data, clinical measures, and biomarker data into useful disease score models

SPACE: Severe Paediatric Asthma Collaborative in Europe; UBIOPRED: Unbiased Biomarkers in Prediction of Respiratory Disease Outcomes; GAN: Global Asthma Network; PERMEABLE: Personalized Medicine Approach for Asthma and Allergy Biologicals Selection; VIRASTHMA2: Inflammatory and Immune Profiles During a Severe Exacerbation in Preschool Asthmatic Children; COBRAPed: Pediatric Cohort of Bronchial Obstruction and Asthma; SysPharmPedia: Systems Pharmacology Approach to Difficult-to-Treat Pediatric Asthma; SHARP: Severe Heterogeneous Asthma Registry, Patient-Oriented; QoL: quality of life.

researchers and patient representatives [38, 39] are needed to address the needs and beliefs of patients and their caregivers. A young patient working group, as currently being established within 3TR, is a great first step to including the patients' voice in treatment considerations.

\section{Next steps}

Collaboration is essential to address the unmet clinical needs and priorities of paediatric severe asthma patients. Joining forces will help bring the field forward and pave the way for a new joint effort to optimise treatment for this vulnerable patient population. This requires a broad engagement to represent all stakeholders and involvement of national respiratory societies and patients to ensure optimal implementation of the research findings into clinical practice. As such, we aim to establish a European working group on joint research and clinical protocols, establish a common European database for paediatric asthma patients, and obtain research funding together. Discussions are ongoing, and we are now reaching out to clinicians, scientists, technology, regulators, healthcare providers and, most importantly, patients into collaborative efforts. This initiative is open for new collaborators to help lead the way for precision medicine of severe paediatric asthma. 
Provenance: Submitted article, peer reviewed.

Conflict of interest: K. Golebski received funds from STIMAG and from the Amsterdam Infection \& Immunity Institute. L.H.M. Dankelman has nothing to disclose. S. Björkander has nothing to disclose. K. Bønnelykke has nothing to disclose. P. Brinkman has nothing to disclose. A. Deschildre has nothing to disclose. Y.E. van Dijk has nothing to disclose. L. Fleming reports grants from Asthma UK, and speakers fees or fees for expert consultation from Teva, AstraZeneca, Sanofi, Respiri and Novartis; all fees paid directly to her institution and outside the submitted work. J. Grigg reports personal fees from GSK, AstraZeneca and Novartis during the conduct of the study. E. Hamelmann has nothing to disclose. S. Hashimoto has nothing to disclose. M. Kabesch has nothing to disclose. S. Klevebro has nothing to disclose. A-H. Maitland-van der Zee has received research grants outside the submitted work from GSK, Boehringer Ingelheim and Vertex; she is the principal investigator of a P4O2 (Precision Medicine for more Oxygen) public-private partnership sponsored by Health Holland involving many private partners that contribute in cash and/or in kind (Boehringer Ingelheim, Breathomix, Fluidda, Ortec Logiqcare, Philips, Quantib-U, Smartfish, SODAQ, Thirona, TopMD and Novartis); and she has served in advisory boards for AstraZeneca, GSK and Boehringer Ingelheim with money paid to her institution. S.K. Merid has nothing to disclose. A. Nieto reports grants and personal fees from Novartis outside the submitted work. J. Niggel has nothing to disclose. C. Nilsson has nothing to disclose. U. Potocnik reports grants from the Ministry of Education, Science and Sport. G. Roberts reports grants from the EU during the conduct of the study. F. Rusconi has nothing to disclose. S. Saglani has nothing to disclose. E. Valente has nothing to disclose. C. van Drunen has nothing to disclose. G. Wang has nothing to disclose. E. Melén reports personal fees from AstraZeneca, Chiesi, Novartis and Sanofi (advisory board activities) outside the submitted work. S.J.H. Vijverberg reports grants from ZonMW during the conduct of the study.

Support statement: The PERMEABLE consortium is supported by ZonMW (project number: 456008004), the Swedish Research Council (project number 2018-05619), the Ministry of Education, Science and Sport of the Republic of Slovenia (contract number C3330-19-252012), and the German Ministry of Education and Research (BMBF) (project number FKZ01KU1909A), under the frame of the ERA PerMed JTC 2018 Call. Funding information for this article has been deposited with the Crossref Funder Registry.

\section{References}

1 Odling M, Andersson N, Ekstrom S, et al. Characterization of asthma in the adolescent population. Allergy 2018; 73: 1744-1746.

2 Lai CKW, Beasley R, Crane J, et al. Global variation in the prevalence and severity of asthma symptoms: Phase Three of the International Study of Asthma and Allergies in Childhood (ISAAC). Thorax 2009; 64: 476-483.

3 Vijverberg SJH, Brinkman P, Rutjes NWP, et al. Precision medicine in severe pediatric asthma: opportunities and challenges. Curr Opin Pulm Med 2020; 26: 77-83.

4 Golebski K, Kabesch M, Melen E, et al. Childhood asthma in the new omics era: challenges and perspectives. Curr Opin Allergy Clin Immunol 2020; 20: 155-161.

5 Pavord ID, Beasley R, Agusti A, et al. After asthma: redefining airways diseases. Lancet 2018; 391: 350-400.

6 Pijnenburg MW, Fleming L. Advances in understanding and reducing the burden of severe asthma in children. Lancet Respir Med 2020; 8: 1032-1044.

7 Lejeune S, Deschildre A, Le Rouzic O, et al. Childhood asthma heterogeneity at the era of precision medicine: Modulating the immune response or the microbiota for the management of asthma attack. Biochem Pharmacol 2020; 179: 114046.

8 Corren J, Kavati A, Ortiz B, et al. Efficacy and safety of omalizumab in children and adolescents with moderate-to-severe asthma: A systematic literature review. Allergy Asthma Proc 2017; 38: 250-263.

9 Gupta A, Ikeda M, Geng B, et al. Long-term safety and pharmacodynamics of mepolizumab in children with severe asthma with an eosinophilic phenotype. J Allergy Clin Immunol 2019; 144: 1336-1342.

10 Bleecker ER, Wechsler ME, FitzGerald JM, et al. Baseline patient factors impact on the clinical efficacy of benralizumab for severe asthma. Eur Respir J 2018; 52: 190936.

11 Agache I, Beltran J, Akdis C, et al. Efficacy and safety of treatment with biologicals (benralizumab, dupilumab, mepolizumab, omalizumab and reslizumab) for severe eosinophilic asthma. A systematic review for the EAACI Guidelines - recommendations on the use of biologicals in severe asthma. Allergy 2020; 75: 1023-1042.

12 Henriksen DP, Bodtger U, Sidenius K, et al. Efficacy of omalizumab in children, adolescents, and adults with severe allergic asthma: a systematic review, meta-analysis, and call for new trials using current guidelines for assessment of severe asthma. Allergy Asthma Clin Immunol 2020; 16: 49.

13 Chipps BE, Lanier B, Milgrom H, et al. Omalizumab in children with uncontrolled allergic asthma: review of clinical trial and real-world experience. J Allergy Clin Immunol 2017; 139: 1431-1444. 
14 Makhecha S, Jamalzadeh A, Irving S, et al. Paediatric severe asthma biologics service: from hospital to home. Arch Dis Child 2021; 106: 900-902.

15 Just J, Deschildre A, Lejeune S, et al. New perspectives of childhood asthma treatment with biologics. Pediatr Allergy Immunol 2019; 30: 159-171.

16 Papadopoulos NG, Custovic A, Cabana MD, et al. Pediatric asthma: an unmet need for more effective, focused treatments. Pediatr Allergy Immunol 2019; 30: 7-16.

17 Lovinsky-Desir S. The use of biologic therapies for the management of pediatric asthma. Pediatr Pulmonol 2020; 55: 803-808.

18 Castro M, Corren J, Pavord ID, et al. Dupilumab efficacy and safety in moderate-to-severe uncontrolled asthma. N Engl J Med 2018; 378: 2486-2496.

19 Rabe KF, Nair P, Brusselle G, et al. Efficacy and safety of dupilumab in glucocorticoid-dependent severe asthma. N Engl J Med 2018; 378: 2475-2485.

20 Yancey SW, Ortega HG, Keene ON, et al. Efficacy of add-on mepolizumab in adolescents with severe eosinophilic asthma. Allergy Asthma Clin Immunol 2019; 15: 53.

21 Simon AK, Hollander GA, McMichael A. Evolution of the immune system in humans from infancy to old age. Proc Biol Sci 2015; 282: 20143085.

22 Berger W, Gupta N, McAlary M, et al. Evaluation of long-term safety of the anti-IgE antibody, omalizumab, in children with allergic asthma. Ann Allergy Asthma Immunol 2003; 91: 182-188.

23 Ortega $\mathrm{H}$, Lemiere $\mathrm{C}$, Llanos JP, et al. Outcomes following mepolizumab treatment discontinuation: real-world experience from an open-label trial. Allergy Asthma Clin Immunol 2019; 15: 37.

24 Holguin F, Cardet JC, Chung KF, et al. Management of severe asthma: a European Respiratory Society/ American Thoracic Society guideline. Eur Respir J 2020; 55: 1900588.

25 Chung KF, Wenzel SE, Brozek JL, et al. International ERS/ATS guidelines on definition, evaluation and treatment of severe asthma. Eur Respir J 2015; 43: 343-373.

26 Phipatanakul W, Mauger DT, Sorkness RL, et al. Effects of age and disease severity on systemic corticosteroid responses in asthma. Am J Respir Crit Care Med 2017; 195: 1439-1448.

27 Papadopoulos NG, Custovic A, Deschildre A, et al. Impact of COVID-19 on pediatric asthma: practice adjustments and disease burden. J Allergy Clin Immunol Pract 2020; 8: 2592-9.e3.

28 Shaker M, Briggs A, Dbouk A, et al. Estimation of health and economic benefits of clinic versus home administration of omalizumab and mepolizumab. J Allergy Clin Immunol Pract 2020; 8: 565-572.

29 Melen E, Guerra S, Hallberg J, et al. Linking COPD epidemiology with pediatric asthma care: implications for the patient and the physician. Pediatr Allergy Immunol 2019; 30: 589-597.

30 van Bragt J, Adcock IM, Bel EHD, et al. Characteristics and treatment regimens across ERS SHARP severe asthma registries. Eur Respir J 2020; 55: 1901163.

31 Saglani S, Bush A, Carroll W, et al. Biologics for paediatric severe asthma: trick or TREAT? Lancet Respir Med 2019; 7: 294-296.

32 Eger K, Kroes JA, Ten Brinke A, et al. Long-term therapy response to anti-IL-5 biologics in severe asthma-A real-life evaluation. J Allergy Clin Immunol Pract 2021; 9: 1194-1200.

33 Liu NM, Carlsen KCL, Cunningham S, et al. First analysis of the Severe Paediatric Asthma Collaborative in Europe registry. ERJ Open Res 2020; 6: 00566-2020.

34 Jackson ND, Everman JL, Chioccioli M, et al. Single-cell and population transcriptomics reveal pan-epithelial remodeling in type 2-high asthma. Cell Rep 2020; 32: 107872.

35 Goodman MA, Moradi Manesh D, Malik P, et al. CRISPR/Cas9 in allergic and immunologic diseases. Expert Rev Clin Immunol 2017; 13: 5-9.

36 Bush A. Pathophysiological mechanisms of asthma. Front Pediatr 2019; 7: 68.

37 Santus P, Ferrando M, Baiardini I, et al. Patients beliefs on intravenous and subcutaneous routes of administration of biologics for severe asthma treatment: a cross-sectional observational survey study. World Allergy Organ J 2019; 12: 100030.

38 Groot B, Dedding C, Slob E, et al. Adolescents' experiences with patient engagement in respiratory medicine. Pediatr Pulmonol 2021; 56: 211-216.

39 Supple D, Roberts A, Hudson V, et al. From tokenism to meaningful engagement: best practices in patient involvement in an EU project. Res Involv Engagem 2015; 1: 5. 